\title{
Pesticide Residues in Buffalo and Human Breast Milk of Vegetables and Fruits Farming Community at Northern of Delta in Egypt
}

Moustafa M.S. Abbassy*

Department of Environmental Studies, Institute of Graduate Studies \& Research, Alexandria University, Egypt

\begin{abstract}
This study was carried out to determine the potential occurrence of pesticide residues in the milk of buffalo and women, which exists in vegetables and fruits farming community in Rosetta region Northern of Delta, Egypt. Milk samples were analyzed by gas chromatography-electron capture detector. Halogenated pesticides were selected includes: 1 Organochlorines; Hexachlorocyclohexane isomers $(\alpha, \beta, y-H C H s)$, dichlorodiphenyltrichloroethane $\left(p, p^{\prime}-\right.$ DDT) and its degradates; $p, p^{\prime}-\mathrm{DDD}$ and DDE, and cyclodiene compounds; Aldrin, dieldrin and endrin. 2- Organophosphorous pesticides; Chlorpyrifos, chlorpyrifos methyl and profenofos. 3- Pyrethroids; Cypermethrin and lambda-cyhalothrin. The tested organochlorine pesticides were detected in most of the samples analyzed at lower frequencies and levels ranged from 2.38 to $3.57 \mu \mathrm{g} / \mathrm{kg}$ in buffalo than of human milk $(2.4-5.78 \mu \mathrm{g} / \mathrm{kg})$. Whereas organophosphorous and pyrethroids found at high predominance of frequencies among all the tested compounds in the buffalo milk than in human milk analyzed. Generally, all samples were positive for at least one of the pesticides; Chlorpyrifos, chlorpyrifos, methyl, profenfos or cypermethrin with mean levels ranged from 1.9 to $5.14 \mu \mathrm{g} / \mathrm{kg}$ of the two milk types, except lambdacyhalothrin at below detection limit in all human breast milk samples. Statistically, significant difference and correlation of organochlorines levels (but no significant difference or correlation for organophosphorous and pyrethroids) were found between the two types of milk investigated. The data available for estimation of daily intakes (ADIs) and extraneous and maximum residue limits (ERLs \& MRLs) revealed that these compounds were found to be lower than the safety thresholds level. Results of this study indicated that in spite of the banning much of organochlorine pesticides (rather than the currently applied (e.g., organophosphorous and pyrethroid pesticides), still contaminating the food stuffs, particularly milk.
\end{abstract}

Keywords: Cypermethrin; Organophosphorous pesticides; Human breast milk; Chlorpyrifos

\section{Introduction}

Certain of halogenated organic compounds such as organochlorine pesticides (OCPs) (rather than pyrothroid and organophosphorous pesticides (OPPs) which are significantly applied to agricultural crops) is characterized by their lipophilicity, and environmental persistence. Thus, it bioaccumulate in the adipose tissues of biota, and biomagnified through different food chains $[1,2]$. At all pesticide types, it poses a risk of causing adverse effects to human health and the environment [3].

Many subsequent studies around the world had been carried out for determination of the pesticide residue levels in different milk kinds of buffalo, cow, goat, camel and sheep, as well as human breast milk in developing and developed countries. From these researches in Egypt; [46] found certain organochlorine pesticide residues such as DDT, DDE, dielderin, endrin, heptachlor, heptachlor epoxide and lindane in buffalo milk. These studies summarized that most of the residual pesticides detected were below the respective maximal permissible limits set by international organizations, except [7] found that from five OCPs (alachlor, exchlorobenzene, dieldrin, methxychlor and linadane) and three OPPs (malathion, parathion-methyl and chlorpyrifos) identified in fresh buffalo milk of agro-industrial zone in upper Egypt, lindane and malathion exceeded EU limits. In other countries, China [8,9] and Italy [10] identified and quantified chlorpyrifos, bifenthrin, deltamethrin, lambda cyhalothrin, imidacloprid and carbofuran pesticides in different milk types from localities of Pakistan. Kara et al. [11] investigated the Th presence of Th OPPs; malathion and Th malaoxon residues in Th buffalo and Th cow milk products in Th the Th western Turkey. Aslam et al. [12] quantified some of organochlorine pesticide residues in the buffalo milk samples of India. Regarding the studies which carried out on the human breast milk, [13] detected OCPs (for example, total DDTs) in breast milk of lactating mother at the main levels of $1315,113.7$ and $517.3 \mathrm{ng} / \mathrm{g}$ fat, respectively. Mirani et al. [14] found endosulfan, DDT, chlorpyrifos, profenofos and lambda-cyhalothrin in milk samples of rural female farm workers in Sindh province of Pakistan. Al Antary et al. [15] detected HCHs and DDTs of chlorinated pesticide residues in human breast milk in Jordan districts. In Brazil, Dallegrave et al. [16] identified chlorpyrifos, bifenthrin, cyhalothrin, permethrin, cypermethrin and deltamethrin in fatty foods. Koomson et al. [17] quantified HCB and $\mathrm{P}, \mathrm{P}$ '- DDE in breast milk of lactating wives of farmers in Accra, Ghana at levels of $1.77 \mu \mathrm{g} / \mathrm{kg}$ and $18.21 \mu \mathrm{g} / \mathrm{kg}$, respectively).

Milk monitoring can provide information about the types and quantities of pesticides in the environment as well as in our daily diet. This information is useful to assess potential health risks associated with the contribution of milk consumption for pesticides intake into the human either for babies or during the following age stages. So, determination of environmental chemicals in the common milk types are of broad scientific interest [12]. So this study aimed to investigate the residual levels of pesticides, including representing group of persistent organochlorine compounds beside certain pesticides of organophosphorous and pyrethroids (which are currently applied in agricultural practices) in milk of buffalo and human living in selected

*Corresponding author: Moustafa M. Abbassy, 163 Horreya Avenue Chatby PC 21526, PO Box 832, Institute of Graduate Studies \& Research, Alexandria University, Alexandria, Egypt, Tel: 002-01006778842; E-mail: moustafabbassy@gmail.com

Received December 12, 2016; Accepted February 08, 2017; Published February 11, 2017

Citation: Abbassy MMS (2017)Pesticide Residues in Buffalo and Human Breast Milk of Vegetables and Fruits Farming Community at Northern of Delta in Egypt. J Environ Anal Toxicol 7: 432. doi: 10.4172/2161-0525.1000432

Copyright: @ 2017 Abbassy MMS. This is an open-access article distributed under the terms of the Creative Commons Attribution License, which permits unrestricted use, distribution, and reproduction in any medium, provided the original author and source are credited. 
Citation: Abbassy MMS (2017)Pesticide Residues in Buffalo and Human Breast Milk of Vegetables and Fruits Farming Community at Northern of Delta in Egypt. J Environ Anal Toxicol 7: 432. doi: 10.4172/2161-0525.1000432

Page 2 of 5

rural areas in Egypt. The obtaining results have contributed to evaluate impacts of pesticide residues on the human health.

\section{Materials and Methods}

The sampling was conducted in period of three months (June to August 2015) as intensive vegetable cultivation and fruiting period among the study area. Sixteen milk samples were taken from buffalo and twelve from human lactating mothers living in and nearby certain represented villages of Rosetta, El-Beheara governorate, north of the delta, Egypt. The samples were kindly collected from individual buffalo animals and women (two samples from each source within the sampling period). The tested pesticides include; Organochlorines $\alpha, \beta, \gamma$-HCHs,p, $\mathrm{p}^{\prime}$ - dichlorodiphenyltrichloroethane ( $\left.\mathrm{p}, \mathrm{p}-\mathrm{DDT}\right)$ and its degradates; $\mathrm{p}$, p-DDD and DDE, cyclodiene compounds; Aldrin, dieldrin and endrin), organophosphorous (chlorpyrifos, chlorpyrifos methyl, profenofos) and pyrethroids (cypermethrin and lambda-cyhalothrin). The milk sample (about $60 \mathrm{ml}$ ) was manually expressed from each of the two sources (by the buffalo's owner and by the volunteer lactating women themselves) into clean glass containers pre-rinsed with acetone and n-hexane. The collected samples were kept frozen at $-20^{\circ} \mathrm{C}$ until analysis.

All solvents; n-hexane, acetone and dichloromethane were purchased from Fisher scientific (Loughborough, UK) as a pesticide residue analytical grade. Anhydrous sodium sulphate (granules) and florisil were Sigma-Aldrich (Poole, UK) grade were prepared based on the methods of $[18,19]$. The glassware used for the analysis was rinsed with distilled water followed by acetone and n-hexane before use to prevent contamination. The Procedural blank sample was carried out to assess the purity of all chemicals and glassware used against any interference. Technical pesticides standards ( $\geq 98.0 \%$ of purity) were obtained from Supelco USA. A mixture of the standard components at four different concentrations $(5,10,15,20,40 \mathrm{pg} / \mu \mathrm{l})$ were prepared and applied for calibration of gas chromatography system (which used in this study) prior to and in between each batch samples analysis.

Briefly, duplicate of milk sample ( $30 \mathrm{ml}$ sub-sample of each sample) was extracted for pesticides residue analysis based on the US-EPA (1985) protocol. The sample was extracted with acetone:n-hexane (1:1) mixture, then re-extracted twice. The organic phases were combined, evaporated to about $5 \mathrm{ml}$ using a rotary evaporator, then to $1 \mathrm{ml}$ by gentle stream of nitrogen. The final extract was subjected to a cleanup procedure according to Fillmann et al. [18] by bathing it through florisil column (20 cm length, $1 \mathrm{~cm}$ i.d.) and the analyst eluted using $70 \mathrm{ml}$ of $\mathrm{n}$-hexane followed by a mixture of $70 \% \mathrm{n}$-hexane and $30 \%$ dichloromethane. All eluents were collected altogether, then evaporated to about $5 \mathrm{ml}$ using a rotary evaporator, then to dryness by a gentle stream of nitrogen and re-dissolved in $0.5 \mathrm{ml} \mathrm{n}$-hexane as final extract volume for injection an aliquot ( $\mu \mathrm{l})$ into GC-ECD.

Detection of pesticide residues was achieved by injection of 1 to 2 $\mu \mathrm{l}$ from the final extract using thermo scientific tri-plus auto-sampler into a gas chromatograph (thermo scientific, 2009) equipped with ${ }^{63} \mathrm{Ni}$ micro-electron capture detector $(\mu \mathrm{ECD})$. A fused silica Ultra 2 capillary column; $30 \mathrm{~m}$ x $0.32 \mathrm{~mm} \times 0.25 \mu \mathrm{m}$ film thickness was used. Nitrogen (GC grade) was used as the carrier gas and detector makeup gas at 1 and $35 \mathrm{ml} / \mathrm{min}$ flow rate, respectively. The chromatographic operating conditions were as follows: Splitless injection mode at temperature of $250^{\circ} \mathrm{C}$, detector; $310^{\circ} \mathrm{C}$. Oven program: initial temp; $80^{\circ} \mathrm{C}$, initial hold for $1 \mathrm{~min}$, ramp $1: 4.0^{\circ} \mathrm{C} / \mathrm{min}$ to $160^{\circ} \mathrm{C}$, hold for $1 \mathrm{~min}$, ramp $2: 2.0^{\circ} \mathrm{C} / \mathrm{min}$ to $220^{\circ} \mathrm{C}$, hold for $0.0 \mathrm{~min}$, ramp $3: 10.0^{\circ} \mathrm{C} / \mathrm{min}$ to $280^{\circ} \mathrm{C}$, hold for $5 \mathrm{~min}$. Retention time and area of the resolved peaks were used as the basis for qualitative and quantitative analysis of the analytes, respectively. Residue levels were expressed as $\mu \mathrm{g} / \mathrm{kg}$ milk (ppb). The method detection limits (MDLs) of the tested pesticides were determined according to US-EPA method 608. The obtained limits were found at ranges of 0.080-0.125 $\mu \mathrm{g} / \mathrm{kg}$ milk.

Because the study is subjected for determining the pesticide residues of different chemical groups, which their residues are extracted altogether using one or mixture of two solvents. Therefore, fortification experiment was performed for all target pesticides in order to assess the analytical procedure efficiency. Triplicate buffalo milk sub-samples (30 $\mathrm{ml}$ each) were spiked with $0.5 \mathrm{ml}$ of standard working mixture of the tested pesticides at levels of $40 \mathrm{pg} / \mu \mathrm{l}$, each. Then, the spiked samples as triplicate were subjected for extraction using different solvent systems; (1): $n$-Hexane (2):Acetone followed by a partition into dichloromethane (3):Acetone:n-hexane (1:1). Then, each extract was cleaned-up and injected into GC as the same procedures as for non-spiked milk samples. The recovery percentage for each analyte was determined by comparing the peak area of each compound concentration of the standard working solution with those of spiked into the milk samples.

\section{Statistical analysis}

A Statistical analysis by a two-way analysis of variance (ANOVA) and correlation tests using SPSS software was used to determine if significant correlation of the residual pesticide levels identified between the buffalo and human milk samples analyzed. A value of zero level was assigned for results below the limit of detection (BLD). Data were expressed as means \pm standard error. Values are Interpreted at range of: -1 to 0.0 to 1 (very high negative correlation, little if any negative correlation, very high positive correlation).

\section{Results}

The study was carried out to determine the residual levels of certain chlorinated pesticides of $\alpha, \beta$ and $\gamma$ - HCHs, p, p'- DDT and its degradates; $p, p^{\prime}-D D D$ and DDE, aldrin, dieldrin, endrin, chlorpyrifos, profenfos, cypermethrin and lambda-cyhalothrin in buffalo and human breast milk collected from selected vegetables and horticultural farming area of Rosetta region, north of delta, Egypt.

Among all the tested compounds using the three solvent systems, the recoveries from the spiked buffalo milk samples analyzed were found at percentages averaged from 52 to $97 \%$ of OCs, OPPs and pyrethroid groups (Table 1). For each system of elution, acetone:nhexane (1:1) mixture, and n-hexane, were shown as nearly similar efficient with recoveries of $77.7-90.7 \%$ and $76.7-90 \%$, respectively, while acetone followed by a partition into dichloromethane was at relative lower efficiency of recovery (66-82\%). So, the first (acetone:nhexane (1:1) mixture) was applied in this study for extraction of the three pesticide groups from the field milk samples. All pesticide residue levels quantified in the field milk samples were corrected according to their recovery percentages.

Concerning the tested pesticide residues in buffalo and human breast milk samples, the obtained results listed in Table 2 indicated that residues of the all tested organochlorine pesticides (OCPs) were detected with different frequencies ranged from $26.7 \%$ for aldrin and dieldrin to $80 \%$ for $\alpha-\mathrm{HCH}$ and p,p'-DDE in the buffalo milk, while their frequencies in human milk fluctuated between 50 and $100 \%$ of the total samples analyzed, respectively. Among the OC compounds, the mean levels quantified were at ranges of $1.03 \pm 0.2 \mu \mathrm{g} / \mathrm{kg}$ for aldrin and $2.0 \pm$ $0.7 \mu \mathrm{g} / \mathrm{kg}$ for $\alpha-\mathrm{HCH}$ in the buffalo milk, whereas in human milk the OCs ranged between $1.29 \pm 0.3$ for p,p'-DDD and $2.4 \pm 0.6$ for $\alpha-\mathrm{HCH}$. In general, $\alpha-\mathrm{HCH}$ and $\mathrm{p}, \mathrm{p}^{\prime}-\mathrm{DDE}$ were found at more predominance of 
Citation: Abbassy MMS (2017)Pesticide Residues in Buffalo and Human Breast Milk of Vegetables and Fruits Farming Community at Northern of Delta in Egypt. J Environ Anal Toxicol 7: 432. doi: 10.4172/2161-0525.1000432

Page 3 of 5

\begin{tabular}{|c|c|c|c|c|}
\hline \multirow{2}{*}{ Solvent type } & \multicolumn{3}{|c|}{ Recovery percentages of pesticides (\%) } \\
\cline { 2 - 5 } & Organochlorines & Organphosphorous & Pyrethroids & Averages \\
\hline n-Hexane & $70-88$ & $83-93$ & $77-89$ & $76.7-90$ \\
\hline $\begin{array}{c}\text { Acetone followed by a partition into } \\
\text { dichloromethane }\end{array}$ & $52-79$ & $81-86$ & $65-81$ & $66-82$ \\
\hline n-Hexane:Acetone (1:1) & $85-97$ & $68-84$ & $80-91$ & $77.7-90.7$ \\
\hline
\end{tabular}

Table 1: Recovery percentages (\%) of the tested organochlorine, organophosphorous and pyrethroid pesticides from the Spiked buffalo milk.

*: The Solvent type was selected based on the methods of US-EPA (1984) and Leoni et al. (1992).

\begin{tabular}{|c|c|c|c|c|c|}
\hline \multirow{3}{*}{ Pesticides } & \multicolumn{4}{|c|}{ Pesticide residue levels $(\mu \mathrm{g} / \mathrm{kg})$} & \multirow{3}{*}{$\begin{array}{c}\text { MRLs } \\
\text { (FAO/WHO, 1997, 2004). } \\
{ }^{*} \mathrm{NA}\end{array}$} \\
\hline & \multicolumn{2}{|c|}{ Buffalo milk } & \multicolumn{2}{|c|}{ Human breast milk } & \\
\hline & Mean \pm S.D & Frequency (\%) & Mean \pm S.D & Frequency (\%) & \\
\hline$\alpha-\mathrm{HCH}$ & $2.0 \pm 0.7$ & 80 & $2.4 \pm 0.6$ & 100 & NA \\
\hline$\beta-\mathrm{HCH}$ & $1.58 \pm 0.5$ & 60 & $1.83 \pm 0.4$ & 90 & NA \\
\hline $\mathrm{Y}-\mathrm{HCH}$ & $1.70 \pm 0.7$ & 60 & $1.92 \pm 0.7$ & 90 & 10 \\
\hline$\Sigma-\mathrm{HCHs}$ & $3.57 \pm 1.46$ & 66.7 & $5.78 \pm 1.35$ & 93.3 & - \\
\hline Aldrin & $1.03 \pm 0.2$ & 26.7 & $1.54 \pm 0.3$ & 50 & \multirow{2}{*}{${ }^{*} 6$} \\
\hline Dieldrin & $1.57 \pm 0.6$ & 26.7 & $1.76 \pm 0.4$ & 50 & \\
\hline Endrin & $1.73 \pm 0.4$ & 33.3 & $1.69 \pm 0.6$ & 60 & NA \\
\hline$\Sigma$-Cyclodienes & $1.27 \pm 1.48$ & 28.9 & $2.67 \pm 1.62$ & 53.3 & - \\
\hline$p, p^{\prime}-D D T$ & $1.29 \pm 0.6$ & 46.7 & $1.46 \pm 0.4$ & 70 & 20 \\
\hline$p, p^{\prime}-D D D$ & $1.21 \pm 0.3$ & 40 & $1.29 \pm 0.3$ & 50 & NA \\
\hline$p, p^{\prime}-D D E$ & $2.09 \pm 0.5$ & 80 & $2.32 \pm 0.6$ & 100 & NA \\
\hline$\Sigma-p, p^{\prime}-D D T s$ & $2.74 \pm 1.12$ & 55.6 & $3.98 \pm 1.37$ & 73.3 & - \\
\hline$\Sigma$-OCPs & $7.58 \pm 2.38$ & & $12.44 \pm 2.66$ & & - \\
\hline
\end{tabular}

Table 2: Levels $(\mu \mathrm{g} / \mathrm{kg}$ ) and occurring frequencies (\%) of selected organochlorine pesticides in buffalo and human breast milk in selected rural areas at Rosetta region, north of delta, Egypt, 2015.

* NA-Not Available; ** (Aldrin+Dieldrin)

frequencies and levels among all the tested compounds in the tow milk types analyzed. As a total occurring levels of the tested OC pesticides and its degradates, the study showed that $\Sigma$-cyclodienes and $\Sigma$-p,p'DDTs were found at higher mean levels; $5.78 \pm 1.4$ and $4.49 \pm 1.4 \mu \mathrm{g} /$ $\mathrm{kg}$ in human milk than in buffalo milk; $2.38 \pm 1.2$ and $2.93 \pm 0.9 \mu \mathrm{g} / \mathrm{kg}$, except $\Sigma$-HCHs at $2.4 \pm 0.6$ and $3.57 \pm 1.1 \mu \mathrm{g} / \mathrm{kg}$, respectively.

Table 3 shows the identified residue levels of the tested organophosporous pesticides (chlorpyrifos, chlorpyrifos methyl, profenofos) and pyrethroids (cypermethrin and lambda-cyhalothrin) in the buffalo and human milk analyzed. Chlorpyrifos, chlorpyrifos methyl and profenofos were detected at the mean levels; $3.61 \pm$ $1.2,2.34 \pm 1.0$ and $1.96 \pm 0.3 \mu \mathrm{g} / \mathrm{kg}$ in the buffalo milk, whereas in human milk; $4.4 \pm 1.0,2.66$ and $2.66 \mu \mathrm{g} / \mathrm{kg}$, with the overall mean of $\Sigma$ OPPs at $4.99 \pm 2.6$ and $2.88 \pm 0.3 \mu \mathrm{g} / \mathrm{kg}$ in the two milk types, respectively. Cypermethrin was detected with the frequency of occurrence at $53.3 \%$ and mean levels of $3.94 \pm 1.3 \mu \mathrm{g} / \mathrm{kg}$, while lambda-Cyhalothrin found in $20 \%$ of the total buffalo milk samples analyzed with the mean level of $4.93 \pm 1.0 \mu \mathrm{g} / \mathrm{kg}$. Lambdacyhalothrin was below the detection limits $(0.1 \mu \mathrm{g} / \mathrm{kg}$ milk), while cypermethrin detected in $40 \%$ of the total human milk analyzed at a mean level of $4.4 \pm 1.6 \mu \mathrm{g} / \mathrm{kg}$. As total pyrethroids, it were quantified at mean averages of $5.14 \pm 1.9$ and $4.4 \pm 1.6 \mu \mathrm{g} / \mathrm{kg}$ in the buffalo and human breast milk, respectively.

The statistical analysis for the obtained results (Table 4) revealed that the quantified levels of organochlorine pesticides ( $\mathrm{HCHs}$, cyclodienes, DDTs) are highly significant different and correlated between buffalo and human milk, while no significant variation or correlation found for the levels of organoposphorous and pyrethroid pesticides between the two types of milk samples investigated.

\section{Discussion}

The exposure to OCPs group of persistent organochlorine pollutants (POPs) has been occurred for decades, and levels of these compounds are still to be detected (as the present study) either in buffalo or human milk of agricultural villages or urban regions. Similar finding pattern was showed in the study of Mirani et al. [14]. They found that OCPs (endosulfan, DDT), OPPs (chlorpyrifos, profenofos) and pyrethroids (lambda-cyhalothrin) as major milk pollutants of milk samples of rural female farm workers engaged in cotton, okra, chilies picking, berseem and wheat harvesting activities in Sindh province of Pakistan. Barman et al. [20] found DDT and its degradates; DDD and DDE in cow milk of Dhanbad city, India at levels not exceeded the tolerance levels of WHO/FAO.

The general finding pattern in the present survey is almost similar (but at lower levels) as showed for the other previous studies as in Egypt and Libya $[13,21]$ as well as the reviewed data mentioned and reported [22]. For the other milk types (e.g. cow, buffalo, goat, camel and sheep milk), Ayoub et al. [6]. Dawood et al. [4] identified certain group of organochlorine pesticides in different governorates of Egypt. Shahzadi et al. [10] quantified of organophosphorous (chlorpyrifos), pyrethroid (bifenthrin, deltamethrin and lambda cyhalothrin), insecticide (imidacloprid) and carbamate (carbofuran) pesticides in cow, buffalo, goat, camel and sheeps milk collected from different localities of Lahore, Pakistan. Aslam et al. [12] found that $50 \%$ of the buffalo milk samples of Delhi, India were contaminated with organochlorine pesticides residues. Dallegrave et al. [16] analyzed 17 different pyrethroids and chlorpyrifos in the fat content of animal products, including beef, chicken, eggs, fish, and milk. They found that all samples were positive for at least one of the pesticides; chlorpyrifos, 
Citation: Abbassy MMS (2017)Pesticide Residues in Buffalo and Human Breast Milk of Vegetables and Fruits Farming Community at Northern of Delta in Egypt. J Environ Anal Toxicol 7: 432. doi: 10.4172/2161-0525.1000432

Page 4 of 5

\begin{tabular}{|c|c|c|c|c|c|}
\hline \multirow{3}{*}{ Pesticides } & \multicolumn{5}{|c|}{ Pesticide residue levels $(\mu \mathrm{g} / \mathrm{kg})$} \\
\hline & \multicolumn{2}{|c|}{ Buffalo milk } & \multicolumn{2}{|c|}{ Human breast milk } & \multirow{2}{*}{$\begin{array}{c}\text { MRLs } \\
\text { (FAO/WHO, 2003, } 2013 .\end{array}$} \\
\hline & Mean \pm S.D & Frequency (\%) & Mean \pm S.D & Frequency (\%) & \\
\hline Chlorpyrifos & $3.61 \pm 1.2$ & 40 & $4.4 \pm 1.0$ & 30 & 20 \\
\hline Chlorpyrifos methyl & $2.34 \pm 1.0$ & 26.7 & 2.66 & 10 & 10 \\
\hline Profenofos & $1.96 \pm 0.3$ & 13.3 & 2.66 & 10 & 20 \\
\hline$\Sigma$-Organophosphprous (OPPs) & $2.33 \pm 3.09$ & 26.7 & $2.88 \pm 0.3$ & 16.7 & - \\
\hline Cypermethrin & $3.94 \pm 1.3$ & 53.3 & $4.4 \pm 1.6$ & 40 & 50 \\
\hline Lambda-Cyhalothrin & $4.93 \pm 1.0$ & 20 & "BLD & & 200 \\
\hline$\Sigma$-Pyrethroids & $3.08 \pm 2.97$ & 36.7 & $4.4 \pm 1.6$ & 40 & - \\
\hline
\end{tabular}

Table 3: Levels $(\mu \mathrm{g} / \mathrm{kg})$ and occurring frequencies $(\%)$ of selected organophosphorous and pyrethroid pesticides in buffalo and human breast milk in selected rural areas at Rosetta region, north of delta, Egypt, 2015.

*: BLD-Below the limits of detection (0.080-0.125 $\mu \mathrm{g} / \mathrm{kg} \mathrm{milk).}$

\begin{tabular}{|c|c|c|c|c|c|c|c|c|c|c|}
\hline & $\Sigma-\mathrm{HCHs}^{*}(\mathrm{~B})$ & $\begin{array}{l}\Sigma \text {-Cyclo-dienes } \\
\text { (B) }\end{array}$ & $\begin{array}{c}\sum-p, p^{\prime}- \\
\text { DDTs (B) }\end{array}$ & $\sum$-OCPs (B) & $\Sigma-\mathrm{HCHs}^{*}(\mathrm{H})$ & $\begin{array}{l}\sum \text {-Cyclo- } \\
\text { dienes }(\mathrm{H})\end{array}$ & $\begin{array}{c}\Sigma-p, p^{\prime}-\text { DDTs } \\
(H)\end{array}$ & $\begin{array}{c}\sum \text {-OCPs } \\
(\mathrm{H})\end{array}$ & $\begin{array}{l}\text { OPPs } \\
\text { (B) }\end{array}$ & $\begin{array}{l}\text { pyrthroids } \\
\text { (B) }\end{array}$ \\
\hline$\Sigma-\mathrm{HCHs}^{*}(\mathrm{~B})$ & 1 & & & & & & & & & \\
\hline$\Sigma$-Cyclo-dienes (B) & 0.1 & 1 & & & & & & & & \\
\hline$\Sigma-p, p^{\prime}-$ DDTs (B) & 0.16 & -0.46 & 1.00 & & & & & & & \\
\hline$\sum$-OCPs (B) & 0.81 & 0.58 & 0.15 & 1.00 & & & & & & \\
\hline$\Sigma-\mathrm{HCHs}^{*}(\mathrm{H})$ & 0.51 & 0.44 & -0.38 & 0.52 & 1.00 & & & & & \\
\hline$\sum$-Cyclo-dienes $(\mathrm{H})$ & 0.38 & 0.51 & -0.63 & 0.38 & 0.36 & 1.00 & & & & \\
\hline$\Sigma-p, p^{\prime}-$ DDTs $(H)$ & 0.26 & 0.02 & 0.67 & 0.43 & -0.05 & -0.14 & 1.00 & & & \\
\hline$\sum$-OCPs $(\mathrm{H})$ & 0.62 & 0.55 & -0.23 & 0.72 & 0.70 & 0.72 & 0.40 & 1.00 & & \\
\hline organophosphorous (B) & 0.35 & -0.29 & 0.86 & 0.34 & -0.32 & -0.30 & 0.71 & 0.02 & 1.00 & \\
\hline pyrthroids (B) & -0.32 & -0.33 & 0.58 & -0.24 & -0.33 & -0.56 & 0.41 & -0.30 & 0.50 & 1.00 \\
\hline organophosphorous $(\mathrm{H})$ & -0.45 & -0.22 & -0.11 & -0.50 & -0.20 & -0.15 & -0.61 & -0.51 & -0.30 & 0.14 \\
\hline pyrthroids $(\mathrm{H})$ & -0.30 & 0.34 & 0.01 & 0.02 & 0.28 & -0.23 & -0.13 & -0.06 & -0.06 & 0.25 \\
\hline
\end{tabular}

Table 4: Correlation pattern between the organochlorine (OCP), organophosphorous (OPP) and pyrethroid pesticide levels in buffalo and human breast milk of selected rural areas at Rosetta region, north of delta, Egypt, 2015.

*: (B) Buffalo, $(\mathrm{H})$ Human

- Values are Interpreted at range of:-1-0.0-1 (very high negative correlation-Little if any negative correlation-very high positive correlation).

bifenthrin, cyhalothrin, permethrin, cypermethrin, or deltamethrin, with mass fraction levels ranging from 0.03 to $270 \mathrm{ng} \mathrm{g}^{-1}$ lyophilized sample. Jawaid et al. [23] found pesticide residues of chloripyrifos, a and $\beta$ endosulfan, profenofos and bifenthrin in raw milk at high levels and violated the MRL set by the FAO/WHO as compared to processed milk.

In the present study, the occurrence pattern of the tested pesticides that might be refers to the nature of feeding style of the mammals through the different food chains related to the transformation, bioaccumulation and biomagnification of such persistent OCs compared with the more degradable pesticides such as OPPs and pyrethroids which are relatively at high levels and frequencies in omnivores than of herbivore biota. On the other side, the direct exposure of buffalo to many of pesticide residues via its feeding on contaminated fodder (e.g. grasses, maize, clover) that adjacent to the sprayed vegetables and fruits fields, also due to exposure from contaminated buffalo drinking water of irrigated canals and drains. Whereas the potential sources for milk contamination of the lactating woman living in or near the investigated areas might be refers to their consumption on food contaminated with the residues of currently applied pesticides, or due to sharing of some lactating women for the field practices with their family. Another reason for women exposure might be occasionally, resulting from using domestic aerosols for home pest control. The statistical analysis for the obtained results is for somewhat supports the distribution pattern of these results, where the detected levels of organochlorine pesticides (HCHs, cyclodienes, DDTs) are highly significant different and correlated between buffalo and human milk, while no significant variation or correlation found for the levels of organoposphorous and pyrethroid pesticides between the two types of milk samples investigated.

Regarding extraneous residue limits of pesticide residues in buffalo milk (ERLs), assuming that a person weighing $60 \mathrm{~kg}$ consumes two glasses of milk per day and night (about $330 \mathrm{~g}$ ), the average daily intake of pesticides detected was calculated and compared with the accepted daily intake data reported by $[24,25]$. It was found that the levels of each pesticides studied are below the recommended limits.

FAO/WHO [26] reported that daily breastfeeding intake of human milk by an infant consume $500-800 \mathrm{ml}$ with average of $120 \mathrm{~g} / \mathrm{kg}$ body weight day with an average of $5.7-7.3 \mathrm{~kg}$ baby weight an average. Estimation of daily intake (EDI) of pesticides analyzed in this study was calculated and compared with international limits. The corresponding ADIs of the tested pesticides by breast-fed infants were generally below (as bufallo milk) the established ADIs by FAO/WHO [25,27].

\section{Conclusion}

It can be concluded from the present study that most of the tested organochlorine compounds were detected at high occurring frequencies in both buffalo and human breast milk samples analyzed. Statistically, significant differences and correlation of OCP levels (but not for the OPPs or pyrethroids) between the two investigated milk types. These findings clearly indicate that OCPs are bio-concentrated at similar mode either in buffalo or human, rather than in the other tested pesticides investigated. We can recommend that preventive measures, 
Citation: Abbassy MMS (2017)Pesticide Residues in Buffalo and Human Breast Milk of Vegetables and Fruits Farming Community at Northern of Delta in Egypt. J Environ Anal Toxicol 7: 432. doi: 10.4172/2161-0525.1000432

such as food monitoring and dietary intake assessment need to be adopted in order to reduce the body burden of the pesticide residues.

\section{Acknowledgement}

I thank husbands of the women lactating mothers who donated their breast milk, and also grateful to the farmers who provided the buffalo milk samples. appreciate all for their good understanding the study aim and providing the samples.

\section{References}

1. Kampire E, Kiremire BT, Nyanzi SA, Kishimba M (2011) Organochlorine pesticide in fresh and pasteurized cow's milk from Kampala markets. Chemosphere 84: 923-927.

2. Iftikhar B, Siddiqui S, Rehman S (2014) Assessment of the dietary transfer of pesticides to dairy milk and its effect on human health. African $\mathrm{J}$ of Biotechnol 13: $476-485$

3. Hoekstra PF, Hara O, Fisk TM, Borgå AT, Solomon K, et al. (2003) Trophic transfer of persistent organochlorine contaminants (OCs) within an Arctic marine food web from the southern Beaufort-Chukchi Seas. Environ Pollut 124:509-522.

4. Dawood AA, Helal MA, Mohamed SA, Ali WH (2004) Detection of organochlorine pesticide residues in samples of cow milk collected from Sohag and Qena governorates. Ass Univ Bull Environ Res 7: 105-116.

5. Ahmed NS, Zaki EM (2009) Detection of Some Organochlorine Pesticides in raw Milk in Giza Governorate. J of Appl Sci Res 5: 2520-2523.

6. Ayoub MM, Desoki ME, Hassanin AS, Thabet WM, Mansour MH, et al. (2012) Detection of pesticide residues in milk and some dairy products. J Plant Prot and Path Mansoura Univ 3: 865-880.

7. Shaker EM, Elsharkawy EE (2015) Organochlorine and organophosphorus pesticide residues in raw buffalo milk from agroindustrial areas in Assiut, Egypt. Environ Toxicol Pharmacol 39: 433-440.

8. Qu W, Suri RP, Bi X, Sheng G, Fu J (2010) Exposure of young mothers and newborns to organochlorine pesticides (OCPs) in Guangzhou, China. Sci Total Environ 408: 3133-3138.

9. Guerranti C, Palmieri M, Mariottini M, Focardi SE (2011) Persistent organic pollutants in human milk from central Italy:levels and time trends-FAO/WHO maximum residue limits in codex Alimentarious. Toxicology 1-6.

10. Shahzadi N, Imran M, Sarwar M, Hashmi AS, Wasim M (2013) Identification of pesticides residues in different samples of milk. $\mathrm{J}$ of Agroalimentary Processes and Technol 19: 167-172.

11. Kara R, Ince S (2016) Evaluation of Malathion and Malaoxon Contamination in Buffalo and Cow Milk from Afyonkarahisar Region, Turkey, Using Liquid Chromatography/Tandem Mass Spectrometry-a Short Report. Pol J Food Nutr Sci 66: 57-60.

12. Aslam M, Rais S, Alam M (2013) Quantification of Organochlorine Pesticide Residues in the Buffalo Milk Samples of Delhi City, India. J of Environ Protec 4: 964-974.
13. Abd Al-Rahman SH (2012) Persistent organochlorines in human breast milk from Al-Sharkia Governorate, Egypt. Int J of Environ Anal Chem 92: 12151225.

14. Sheikh SA, Mirani BN, Panhwar AA, Nizamani SM, Mastoi S (2014) Pesticide residues composition of milk samples of female farm workers Saghir Sheikh. Int $\mathrm{J}$ of Res. 1: 1627-1633.

15. Al Antary TM, Alawi MA, Estityah H, Haddad N, Al-Antary ET (2015) Chlorinated pesticide residues in human breast milk collected from southern Jordan in 2012/2013. Toxin Rev 34: 190-194.

16. Dallegrave A, Pizzolato TM, Barreto F, Eljarrat E, Barceló D (2016) Methodology for trace analysis of 17 pyrethroids and chlorpyrifos in foodstuff by gas chromatography-tandem mass spectrometry. Anal Bioanal Chem 408 : 7689-7697.

17. Koomson CK, Owusu EO, Obeng-Ofori D, Kingsford-Adaboh R (2016) Residual assessment of Insecticides in body fluids (Breast Milk), crops and the ecosystem in Horticultural farming Communities in Accra, Ghana. $\mathrm{J}$ of Dyn in Agri Res 3: 1-11.

18. Fillmann G, Readman JW, Tolosa I, Bartocci J, Villeneuve JP, et al. (2002) Persistent organochlorine residues in sediments from the Black Sea. Mar Pollut Bull 44: 122-133.

19. US-EPA Method 608 (1984) Organochlorine Pesticides and PCBs. EPA 600/4 84-061, National Technical Information Service, PB84-211358, Springfield, Virginia 22161

20. Barman RM, Mishra BK, Barman A (2016) OCP Residues Analysis in Cow Milk of Dhanbad City, Jharkhand, India. Int $\mathrm{J}$ of Applied and Pure Sci and Agri 02: 1-11.

21. Zeinab HM, Refaat GA, El-Dressi AY (2011) Organochlorine pesticide residues in human breast milk in El-Gabal Al-Akhdar, Libya. Int Conf on Life Sci and Technol 3:146-149.

22. Akhtar S, Ahad K (2016) Status of Pesticides Residues in Milk and Milk Products: A Review. Austin J of Nutri and Food Sci 4: 1-6.

23. Jawaid S, Talpur FN, Nizamani SM, Khaskheli AA, Afridi HI (2016) Multipesticide residue levels in UHT and raw milk samples by GC- $\mu$ ECD after QuEChER extraction method. Environ Monitoring and Assessment. 188: 230-239.

24. FAO/WHO (1993) Portion of Commodities to which Codex MRLs apply in Codex Alimentarius, 2nd ed., Vol 2. Pesticide Residues, Section 4.1. Joint FAO/WHO Food Standard Programme. FAO Rome.

25. FAO/WHO (1994) Codex Alimentarius Commission. CX/2-94, Joint FAO/WHO Food Standards Programme, Codex Committee on Pesticide Residues, FAO/ WHO, Roma.

26. FAO/WHO (1985) The quantity and quality of breast milk. Report of the WHO collaborative study on breast feeding. WHO Geneva, Switzerland.

27. FAO/WHO (1996) Codex Alimentarius. Pesticide Residues in Food-Joint FAO/ WHO Food Standards Programme. Codex Alimentarius Commission, FAO/ WHO, Roma. 